\title{
Eficiencia de uso de nutrientes en ají tabasco (Capsicum frutescens $L$.) y habanero (Capsicum chínense Jacq)
}

\section{Efficiency of use of nutrients in hot pepper tabasco (Capsicum frutescens L.) and habanero (Capsicum chínense Jacq)}

\author{
Eficiência de uso de nutrientes na pimenta tabasco \\ (Capsicum frutescens L.) e "habanero" (Capsicum \\ chínense Jacq)
}

\author{
María del Pilar Romero-Lozada1, Christian Felipe Enciso Murillo², Sandra Marcela Garcia ${ }^{3}$, \\ Juan José Wagner Guerrero ${ }^{4}$, Yina Jazbleidi Puentes-Páramo ${ }^{5}$ \& Juan Carlos Menjivar-Flores ${ }^{6}$ \\ ${ }^{1}$ Ingniera Agrónoma, Estudiante de Maestría en Ciencias Agrarias. 2, 3y 4 Estudiante de Ingeniería Agronómica. \\ ${ }^{5}$ Ingeniera Agrónoma, Magister en Biotecnología de plantas, Doctora en Ciencias Agropecuarias. \\ ${ }^{6}$ Ingeniero Agrónomo, Magister en Suelos y Aguas, Doctor en Ciencia del Suelo.

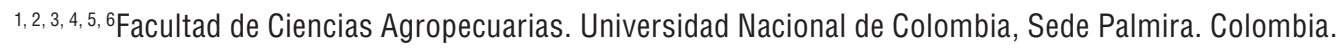 \\ 1,2,3,4mpromerol@unal.edu.co, 5yjpuentesp@unal.edu.co, ${ }^{5} j \mathrm{cmenjivarf@unal.edu.co}$
}

\section{Resumen}

El manejo adecuado de la nutrición de un cultivo implica hacer un uso eficiente de los nutrientes. Por tanto, esta investigación tuvo como objetivo determinar la eficiencia agronómica (EA) y de recuperación del fertilizante (ERF) para nitrógeno, fósforo y potasio en ají habanero y ají tabasco y su influencia en el rendimiento. El diseño experimental consistió en bloques completos al azar con cuatro tratamientos y cuatro repeticiones. Los tratamientos fueron T1: Testigo, T2: fertilización según el programa que maneja el Centro Experimental de la Universidad Nacional de Colombia (CEUNP) para N-P-K, T3: programa de fertilización de CEUNP + 50\% y el T4: programa de fertilización de CEUNP $+100 \%$. Para ají tabasco (C. frutescens L.) y habanero (C. chinense J.) el análisis de varianza dio diferencias altamente significativas $(p<0,001)$ por efecto de los tratamientos tanto para la eficiencia agronómica (EA) como de recuperación del fertilizante (ERF). Los resultados muestran que los mayores valores de EA y ERF para N-P-K en tabasco se presentaron en el tratamiento $\mathrm{T} 4$, y en habanero se presentaron en el tratamiento T3, excepto para la ERF de fósforo. Así, estos permiten observar que ají tabasco presenta bajas eficiencias agronómicas y de recuperación de nutrientes con respecto al tipo habanero. Los mejores rendimientos de tabasco y habanero se presentan en el T4 y los menores en el T1, lo que sugiere una clara influencia del genotipo.

Palabras clave: fertilidad del suelo, fertilización, nutrición mineral, rendimiento. 


\section{Abstract}

The proper handling of a crop nutrition involves efficient use of nutrients. Therefore, this research had as objective determine the efficiency agronomic (EA) and of recovery of the fertilizer (ERF) for nitrogen, phosphorus and potassium in Chili habanero and pepper tabasco and its influence in the performance. The experimental design consisted of complete blocks at random with four treatments and four replications. The treatments were T1: witness, T2: fertilization according to the program that manages the Experimental Center of the National University of Colombia (CEUNP) for n-p - K, T3: fertilization of CEUNP 50\% and T4 program: CEUNP $100 \%$ fertilization program. For pepper tabasco (C. frutescens L.) and habanero (C. chinense J.) the analysis of variance gave differences highly significant ( $p$ \&lt; 0.001) by effect of them treatments both for the efficiency agronomic (EA) as of recovery of the fertilizer (ERF). The results show that the highest values of $E A$, and ERF for $n-p-K$ in tabasco were presented in the T4 treatment, and habanero presented in the T3 treatment, except for the ERF of phosphorus. Thus, these enable observe that tabasco chili presents low efficiencies agronomic and recovery of nutrients to the habanero type. The best yields of tabasco and habanero are presented in the T4 and minors in T1, which suggests a clear influence of genotype.

Key-words: fertilization, mineral nutrition, soil fertility, yield.

\section{Resumo}

O manejo adequado da nutrição de uma cultura implica fazer uso eficiente dos nutrientes. Por isso, este trabalho de pesquisa teve como objetivo determinar a eficiência agronômica (EA) e de recuperação de fertilizante (ERF) para nitrogênio, fósforo e potássio em pimenta "habanero" e tabasco e sua influência no rendimento. $O$ delineamento experimental foi de blocos ao acaso com quatro tratamento e quatro repetições. O tratamentos foram os seguintes T1: Controle, T2: fertilização recomendada pelo Centro Experimental da Universidad Nacional de Colombia (CEUNP) para N-P-K, T3: programa de fertilização de CEUNP + 50\% e T4: programa de fertilização de CEUNP + 100\%. Para pimenta tabasco (C. frutescens L.) e "habanero" (C. chinense J.) a análise de variância mostrou diferenças significativas $(p<0,001)$ devido ao efeito dos tratamentos tanto para EA quanto para ERF. Os resultados mostram que maiores valores de EA e ERF para N-P-K em tabasco foram apresentados no tratamento T4 e em "habanero" no tratamento T3, com exceção de ERF de fósforo. Assim, é possível observar que a pimenta tabasco apresenta baixas eficiências agronômicas e de recuperação de nutrientes quando comparada com o tipo "habanero". Os melhores rendimentos de tabasco e "habanero" foram observados no T4 e o mais baixo no T1 sugerindo uma influência marcada do genótipo.

Palavras-chave: fertilidade do solo, fertilização, nutrição mineral, rendimento.

\section{Introducción}

Diversos factores pueden afectar el desarrollo del cultivo de ají (Capsicumspp.) y por ende su productividad, tales como la falta o exceso de fertilización y el déficit hídrico, entre otros, lo cual se traduce en importantes reducciones del rendimiento (Ismail, 2010); en el mismo sentido, Puentes, Menjívar \& Aranzazu (2014) afirman que la producción no solo depende de la fertilidad del suelo, sino de factores relacionados con la genética de la planta y el medio ambiente, es decir, la interacción de ambos. Un manejo adecuado de la nutrición del cultivo implica hacer un uso eficiente de nutrientes (UEN), en ese orden de ideas, Marouani \& Harbeoui (2016) sugieren que la eficiencia en el uso de nutrientes se relaciona con incrementos en la absorción de nutrientes y reducción de las pérdidas, así mismo, Baligar, Fageria \& He (2001) consideran que el UEN disminuye la pérdida de 
nutrientes, mejora el rendimiento y calidad de los cultivos, lo cual indirectamente, se traduce en una reducción de los costos por uso de fertilizantes, y en una protección del medio ambiente al evitar el uso excesivo de fertilizantes (Zhu et al., 2005). Por otra parte, González et al. (2016) reportan que el uso eficiente de nutrientes se afecta con altas dosis de fertilización y textura del suelo, en este sentido, es importante y necesario conocer las eficiencias para cada cultivo o variedad por zona.

Las investigaciones en ají Capsicum.spp, ya sean de tipo habanero o tabasco, se han enfocado en obtener variedades o líneas con altos rendimientos y concentración de capsaicina, en función de la aplicación de fertilizantes o inoculantes microbianos, de las densidades de siembra y de las aplicaciones de diferentes láminas de riego, entre otras (Reyes et al., 2014, Borges et al., 2010, Díaz et al., 1999, Gil et al., 2012, Quintal et al., 2012). Así mismo, se han determinado los requerimientos nutricionales, más no hay resultados sobre el uso eficiente de nutrientes; es así, que los productores no saben cuánto kg de ají producen por cada $\mathrm{kg}$ de nutriente aplicado, mucho menos cuánto de lo que se aplica al suelo es recuperado por el cultivo, y por esta razón se utilizan iguales dosis nutricionales para cualquier tipo de ají, sea tabasco, habanero, u otro.

En ese orden de ideas, la presente investigación buscó determinar el uso eficiente de nutrientes (UEN) para nitrógeno $(\mathrm{N})$, fósforo $(\mathrm{P})$ y potasio $(K)$,en términos de la eficiencia agronómica (EA) y eficiencia de recuperación del nutriente (ERN) de dos líneas de ají elite de la Universidad Nacional de Colombia seleccionadas para alta capsaicina, correspondientes a Capsicum frutescens tipo tabasco y Capsicum chínense Jacq tipo habanero.

\section{Materiales y métodos}

La investigación se realizó en el Centro Experimental de la Universidad Nacional de Colombia, sede Palmira (CEUNP), ubicado en el municipio de Candelaria (vereda El Carmelo) en el departamento del
Valle del Cauca, Colombia, a 980 msnm, con temperatura promedio de $24{ }^{\circ} \mathrm{C}$, humedad relativa del $75 \%$ y coordenadas $3^{\circ} 24^{\prime}$ latitud norte y $76^{\circ} 26^{\prime}$ longitud oeste.

Al inicio de la investigación se muestreó el suelo a una profundidad entre $20-30 \mathrm{~cm}$ para evaluar sus propiedades físicas y químicas, las cuales se determinaron en el laboratorio de suelos del Centro Internacional de Agricultura tropical CIAT y se interpretaron los resultados con valores de referencia del Instituto Colombiano Agropecuario (ICA, 1992); el pH se determinó por el método del potenciómetro 1:1; materia orgánica (M.O) por Walkley-Black; fósforo por el método de Olsen Espectrometría UV; calcio, magnesio, sodio, potasio y capacidad de intercambio catiónico $(\mathrm{CIC})$ por acetato de amonio pH 7,0, cuantificado por absorción atómica; boro por el método de agua caliente (Espectrometría y Azometina), densidad aparente por el método del núcleo y textura por Bouyoucus.

Se utilizó un diseño experimental en bloques completamente al azar (BCA), con 4 tratamientos y 4 repeticiones para un total de 16 unidades experimentales. La unidad experimental consistió en una parcela de 5,2 $\mathrm{m}$ de ancho por $9 \mathrm{~m}$ de largo, en donde la distancia entre plantas fue de $0,60 \mathrm{~m}$ y entre surcos de 1,3 m. En cada surco se sembraron 15 plantas, y se cosecharon los dos surcos centrales.

Los tratamientos evaluados fueron T1: Testigo, T2: Fertilización según el programa que maneja el Centro Experimental de la Universidad Nacional de CoIombia CEUNP (N: 150, $\mathrm{P}_{2} \mathrm{O}_{5}: 100$ y $\left.\mathrm{K}_{2} \mathrm{O}: 300\right)$, T3: programa de fertilización de CEUNP $+50 \%$ y el T4: programa de fertilización de CEUNP + 100\%. Las cantidades de fertilizante se fraccionaron en seis aplicaciones de acuerdo a la etapa de desarrollo del cultivo, hasta los 90 días (Tabla 1). 
Tabla 1. Porcentaje de fertilización por etapa de desarrollo del cultivo de ají.

\begin{tabular}{l|c|c|c}
\hline ETAPA DE DESARROLLO & $\mathbf{N}(\%)$ & $\mathbf{P}_{2} \mathrm{O}_{5}(\%)$ & $\mathbf{K}_{2} \mathrm{O}(\%)$ \\
\hline ETAPA 1 (10 DDT) & 10 & 30 & 10 \\
\hline ETAPA 2 (25 DDT) & 20 & 25 & 15 \\
\hline ETAPA 3 (45 DDT & 30 & 20 & 20 \\
\hline ETAPA 4 (70 DDT) & 20 & 15 & 20 \\
\hline ETAPA 5 (85 DDT) & 10 & 10 & 20 \\
\hline ETAPA 6 (90 DDT) & 10 & - & 15 \\
\hline TOTAL (\%) & 100 & 100 & 100 \\
\hline
\end{tabular}

DDT: días después del trasplante; $\mathrm{N}$ : nitrógeno; $\mathrm{P}_{2} \mathrm{O}_{5}$ : fósforo; $\mathrm{K}_{2} \mathrm{O}$ : potasio

En frutos se determinó la concentración de nutrientes entre los que se incluyen: $\mathrm{N}, \mathrm{P}, \mathrm{K}, \mathrm{Ca}, \mathrm{Mg}$, $\mathrm{S}, \mathrm{B}, \mathrm{Cu}, \mathrm{Zn}, \mathrm{Fe}$, y $\mathrm{Mn}$; estos análisis se realizaron en el CIAT siguiendo los protocolos para tejido vegetal (Mckean, 1993) para ello se colectaron 20 frutos completamente maduros por línea y tratamiento. A partir de estos datos se determinó la eficiencia agronómica (EA) y eficiencia de recuperación del fertilizante (ERF), según las ecuaciones 1 y 2, respectivamente, propuestas por Baligar, Fageria \& He (2001):

$k g-1)=R(t)-R(c) C N A$

CNt - CNcCNAx100

En donde:

$\mathrm{R}(\mathrm{t})=$ rendimiento de frutos de acuerdo al tratamiento realizado $(\mathrm{kg}) ; \mathrm{R}(\mathrm{c})=$ rendimiento de frutos del tratamiento testigo $(\mathrm{kg}) ; \mathrm{CNA}=$ cantidad del nutriente aplicado por el fertilizante $(\mathrm{kg})$.

$\mathrm{CN}(\mathrm{t})=$ cantidad del nutriente acumulado en el fruto de acuerdo al tratamiento realizado; $\mathrm{CN}(\mathrm{c})=$ cantidad del nutriente acumulado en el fruto del tratamiento control.

El rendimiento se obtuvo por el peso de los frutos cosechados de cada línea y tratamiento en 11 cortes o cosechas, luego fue llevado a unidades de kg.ha-1. Los datos fueron sometidos a análisis de varianza y prueba de comparación de medias de Tukey $(p \leq 0,05)$, en el programa Statistical Analysis System (SAS).

\section{Resultados y discusión}

Los análisis químico y físico, indican un $\mathrm{pH}$ de 7,7 clasificado como ligeramente alcalino, materia orgánica $(2,1 \%)$, alto fósforo $(75,46 \mathrm{ppm})$, alto $\mathrm{K}(0,42$ $\mathrm{Cmol} / \mathrm{kg})$, alta CIC $(20,95 \mathrm{Cmol} / \mathrm{kg})$, adecuada densidad aparente $\left(1,2 \mathrm{Mg} / \mathrm{m}^{3}\right)$ y textura (Franco arcilloso), las cuales son adecuadas para el cultivo de ají (Ligarreto, Espinosa \& Méndez, 2004).

\section{Eficiencia agronómica (EA) en ají tabasco y habanero}

Para ají tabasco (C. frutescensL.) y habanero ( $C$. chinenseJ.) el análisis de varianza mostró diferencias altamente significativas $(p<0,01)$ por efecto de los tratamientos. En tanto, los mayores valores de EA para N-P-K en tabasco se presentaron en el tratamiento $\mathrm{T} 4$, mostrando superioridad la EAP con respecto a la EAN y EAK, en este sentido, por cada $\mathrm{kg}$ de nutriente aplicado se aumentó el rendimiento en mayor proporción por $\mathrm{P}$, luego $\mathrm{N}$ y K ; similar comportamiento presentó el tipo habanero. Con respecto a este último, los mayores valores de EA para $\mathrm{N}-\mathrm{P}-\mathrm{K}$ se presentaron en el tratamiento $\mathrm{T} 3$, seguido el T2 y por último el T4 (Tabla 2). 
Tabla 2. Eficiencia agronómica de nitrógeno, fósforo y potasio para tabasco y habanero, por tratamiento.

\begin{tabular}{l|c|c|c|c|c|c|c|c|c}
\hline & \multicolumn{3}{c|}{ EAN(kg kg-1) } & \multicolumn{3}{c|}{ EAP(kg kg-1) } & \multicolumn{3}{c}{ EAK(kg kg-1) } \\
\hline Tipo & T2 & T3 & T4 & T2 & T3 & T4 & T2 & T3 & T4 \\
\hline Tabasco & $15,25 \mathrm{~b}$ & $15,01 \mathrm{c}$ & $17,7 \mathrm{a}$ & $22,88 \mathrm{~b}$ & $22,52 \mathrm{c}$ & $26,50 \mathrm{a}$ & $7,63 \mathrm{~b}$ & $7,51 \mathrm{c}$ & $8,83^{\mathrm{a}}$ \\
\hline Habanero & $43,15 \mathrm{~b}$ & $44,14 \mathrm{a}$ & $38,08 \mathrm{c}$ & $64,72 \mathrm{~b}$ & $66,21 \mathrm{a}$ & $57,11 \mathrm{c}$ & $21,57 \mathrm{~b}$ & $22,07^{\mathrm{a}}$ & $19,04 \mathrm{c}$ \\
\hline
\end{tabular}

EAN: eficiencia agronómica de nitrógeno; EAP: eficiencia agronómica de fósforo; EAK: Eficiencia agronómica de potasio. Valores dentro de la misma fila con letras distintas para cada nutriente por eficiencia agronómica difieren estadísticamente según la prueba de Tukey $(p \leq 0,05)$

Estas eficiencias (EAN, EAP y EAK) de acuerdo con los valores de referencia reportados por Chavarría (2013) para CapsicumannuumL., se consideran bajos para los dos tipos de ají, sin embargo, aji tabasco presenta los valores más bajos, aun en las mejores eficiencias que se muestran en el T4.

En términos generales, la menor EA para N-P-K en ají habanero se presentó en el tratamiento T4, tratamiento que aportó la mayor cantidad de nutrientes, y la mayor EA para N-P-K en el T3, lo que sugiere, que a mayor cantidad de nutriente la producción por unidad del mismo tiende a disminuir. Sin embargo, para tabasco la menor eficiencia se presentó en el T2 para $\mathrm{N}$, T3 para $\mathrm{P}$ y K, y la mayor eficiencia en el T4, sugiriendo que a mayor nutriente la producción por unidad del mismo tiende a aumentar, con lo cual se evidencia que los dos tipos de ají tuvieron diferencias en el uso de nutrientes; similares resultados obtuvieron Puentes et al. (2014), lo que evidencia la influencia del genotipo en el uso eficiente de nutrientes, como lo sugiere Aguilar et al. (2016).

Por lo tanto, ají habanero con menor dosis de nutrientes (T3) presenta las mejores eficiencias agronómicas superando los resultados de tabasco, así, este último no alcanza el $50 \%$ de las EA que presenta habanero, aun cuando esté en la mayor dosis de nutrientes (T4), siendo superadas en un $149 \%(\mathrm{~N}-\mathrm{P})$ y $206 \%(\mathrm{~K})$; en ese sentido, sería conveniente replantear las dosis de nutrientes que se están aplicando para tabasco.

\section{Eficiencia de recuperación del fertilizante (ERF) en ají tabasco y habanero}

El análisis de varianza mostró diferencias altamente significativas $(p<0,01)$ por efecto de los tratamientos en la eficiencia de recuperación de N-P-K para los dos tipos de ají. Las mayores ERF en Tabasco fueron para $\mathrm{N}$ en el tratamiento $\mathrm{T} 4$, seguido del T2 y por último T3; $\mathrm{K}$ es el segundo elemento en presentar la mejor eficiencia de recuperación en el T4, posteriormente en el T3 y T2; P es el elemento que menor eficiencia de recuperación presenta de los tres nutrientes, y al igual que los anteriores, tiene su mayor valor en el T4, seguido del T2 y por último el T3; comportamiento similar exhibe el tipo habanero mostrando la mayor recuperación de fertilizante en el orden N, K y P; sin embargo, habanero presenta la mayor eficiencia para $\mathrm{N}$ en el tratamiento $\mathrm{T} 3$, seguido del T4 y por último el T2; $\mathrm{K}$ en el T3, luego T4 y T2; P en el T2, seguido por el T3 y T4(Tabla 3).

Tabla 3. Eficiencia de recuperación de nitrógeno, fósforo y potasio para tabasco y habanero, por tratamiento.

\begin{tabular}{l|c|c|c|c|c|c|c|c|c}
\hline & \multicolumn{3}{c|}{ ERN (\%) } & \multicolumn{3}{c|}{ ERP (\%) } & \multicolumn{3}{c}{ ERK (\%) } \\
\hline Tipo & T2 & T3 & T4 & T2 & T3 & T4 & T2 & T3 & T4 \\
\hline Tabasco & $33,86 \mathrm{~b}$ & $30,73 \mathrm{c}$ & $46,18 \mathrm{a}$ & $4,88 \mathrm{~b}$ & $4,68 \mathrm{c}$ & $5,98 \mathrm{a}$ & $16,8 \mathrm{c}$ & $19,88 \mathrm{~b}$ & $24,63 \mathrm{a}$ \\
\hline Habanero & $117,51 \mathrm{c}$ & $141,93 \mathrm{a}$ & $122,68 \mathrm{~b}$ & $18,64 \mathrm{a}$ & $17,14 \mathrm{~b}$ & $17,04 \mathrm{c}$ & $63,83 \mathrm{c}$ & $83,3 \mathrm{a}$ & $63,99 \mathrm{~b}$ \\
\hline
\end{tabular}

ERN: eficiencia de recuperación de nitrógeno; ERP: eficiencia de recuperación de fósforo; ERK: eficiencia de recuperación de potasio. Valores dentro de la misma fila con letras distintas para cada nutriente por eficiencia de recuperación difieren estadísticamente según la prueba de Tukey $(p \leq 0,05)$ 
Los resultados permiten observar que tabasco presenta baja eficiencia de recuperación de nutrientes con respecto al tipo habanero, lo que implica mayor aplicación de nutrientes, así, en términos generales se estima para Tabasco, que del $100 \%$ de nutriente aplicado al suelo la planta solo aprovecha $33,86 \%$ del $\mathrm{N}$, indicando que se está perdiendo el $66,14 \%$, sin embargo, este valor de recuperación es considerado alto comparado con el reportado por Zhu et al.(2005); en K se recuperó el $24,63 \%$ y en el caso de $\mathrm{P}$, solo se recuperó el 5,98\%; al comparar los valores obtenidos con los reportados por Chavarría (2013) el K es el único elemento que se encuentra bajo, $\mathrm{N}$ y $\mathrm{P}$ se consideran normales; y para ají habanero, $\mathrm{K}$ es el único que se considera normal, mientras $\mathrm{N}$ y $\mathrm{P}$ se encuentran por encima de los valores reportados por el mismo autor. Para el caso de la ERF en habanero, los valores superan el $100 \%$ de lo aplicado, lo cual evidencia que hubo mayor absorción de nutrientes, debido a la disponibilidad de nutrientes del suelo en el tratamiento sin fertilizar (T1), dada su fertilidad natural.
En tanto, los resultados sugieren que no es conveniente aplicar iguales dosis de nutrientes a diferentes tipos de ají, en este caso tabasco y habanero, ya que su respuesta al uso de nutrientes es diferencial, como lo sugiere Puentes et al.(2014).

\section{Rendimiento para ají tipo tabasco y habanero}

El análisis de varianza mostró diferencias altamente significativas $(p<0,01)$ por efecto de los tratamientos en el rendimiento tanto de tabasco y habanero. Así, estos dos tipos de ají obtuvieron los mejores rendimientos en el T4 y los menores en el T1 (Tabla 4), resultados similares reportaron Marouani \& Harbeoui (2016). El rendimiento obtenido para habanero se encuentra por encima de los valores reportado por Quintal et al. (2012), así mismo, es interesante ver que el tipo habanero supera en un $133 \%$ el rendimiento de tabasco, muy seguramente por influencia del genotipo.

Tabla 4. Rendimiento ( $\left.\mathrm{kg} \mathrm{ha}^{-1}\right)$ de frutos en ají tabasco y habanero.

\begin{tabular}{l|c|c|c|c}
\hline Tipo de ají & T1 & T2 & T3 & T4 \\
\hline Tabasco & $4895 d$ & $7183 c$ & $8273 b$ & $10195 a$ \\
\hline Habanero & $12384 d$ & $18856 c$ & $22316 b$ & $23807 a$ \\
\hline
\end{tabular}

T1: tratamiento uno; T2: tratamiento dos; T3: tratamiento tres; T4: tratamiento cuatro Valores dentro de la misma fila con letras distintas difieren estadísticamente según la prueba de Tukey $(p \leq 0,05)$

En tabasco coincide que en el tratamiento T4 donde se obtuvo el mayor rendimiento, también se obtuvo la mayor eficiencia tanto agronómica como de recuperación del fertilizante, esto indica que con mayor cantidad de fertilizante, tal vez, el rendimiento aumentaría. Para habanero, a pesar de que el mayor rendimiento se obtuvo en el tratamiento $\mathrm{T} 4$, las mayores eficiencias se presentan en el tratamiento T3, sugiriendo que a partir de esta dosis nutricional la planta disminuye la eficiencia en el uso de nutrientes, en este sentido, incrementar la eficiencia de uso de nutrientes en ají tiene que ver con genotipos capaces de utilizar nutrientes con mayor eficiencia, como lo sugiere Marouani \& Harbeoui (2016).

\section{Conclusiones}

El ají tipo habanero es mucho más eficiente en el uso de nutrientes que el tipo tabasco, ya que con igual cantidad de nutrientes produce más $\mathrm{kg}$ de fruto por $\mathrm{kg}$ de fertilizante aplicado al suelo, así mismo, la recuperación del fertilizante N-P-K es mayor en habanero, por tanto, la respuesta al uso de nutrientes es diferencial de acuerdo al genotipo.

En relación al rendimiento es claro que la capacidad de absorción de tabasco y habanero sobrepasa los valores de CEUNP, ya que ambos tipos de ají presentan su mayor rendimiento en el T4, tratamiento de mayor aporte nutricional. 


\section{Agradecimientos}

Los autores agradecen al Grupo de Investigación: "Uso y Manejo de Suelos y Aguas con énfasis en Degradación de Suelos" de la Universidad Nacional de Colombia, sede Palmira, y al CEUNP por toda su colaboración en campo.

\section{Literatura citada}

1. Aguilar, C.C., Salvador E.J.A., Aguilar M.I, Mejía C.J.A., Conde M.V.F. \& Trinidad S.A. (2016). Eficiencia agronómica, rendimiento y rentabilidad de genotipos de maíz en función del nitrógeno. Terra Latinoamericana, 34, 419-429. Chapingo, México: Sociedad mexicana de la ciencia del suelo.

2. Baligar, V. C., Fageria, N. K. \& He, Z. L. (2001).Nutrient use efficiency in plant. Communications in Soil Science and Plant Analysis, 32(7), 921-950.London, UK: Taylor \& Francis Group.

3. Borges, G.L., Barrios, M., Cervantes, C.L., Ruiz, N.J., Soria, F.M., Reyes, O.V. \& Villanueva, C.E. (2010). Capsaicinoides en chile habanero (Capsicum chinense Jacq.) bajo diferentes condiciones de humedad y nutrición. Terra latinoamericana, 28(1), 35-41. Chapingo, México: Sociedad mexicana de la ciencia del suelo.

4. Díaz, L., Viloria de Z, A. \& Arteaga de R, L. (1999). Crecimiento vegetativo del pimentón en función de la densidad de plantas y edad del cultivo. Bioagro, 11(2), 69-73. Barquisimeto, Venezuela: Universidad centroccidental "Lisandro Alvarado" (UCLA).

5. Chavarria, V.A.D (2013). Eficiencia de tres fuentes fertilizantes sobre la producción de chile dulce (Capsicum annum) c.v.Natali y sus curvas de absorción, en la producción de chile dulce en invernadero. Rev. Ingeniería agrícola, Vol. 3(1):29-39.La Habana, Cuba: instituto de investigaciones de ingeniería agrícola.

6. Gil, M.J.A., Montaño, N.J. \& Plaza, R. (2012). Efecto del riego y la cobertura del suelo sobre la productividad de los cultivares de ají dulce. Bioagro 24(2):143148. Barquisimeto, Venezuela: Universidad centroccidental "Lisandro Alvarado" (UCLA).
7. González, T.A., Figueroa V.U., Preciado R.P., Núñez, H.G., Luna O.J., \& Antuna, G.O. (2016).Uso eficiente y recuperación aparente de nitrógeno en maíz forrajero en suelos diferentes. Rev. Mex. Cienc. Agríc., 7(2), 301309.Chapingo, México: Instituto Nacional de Investigaciones Forestales, Agrícolas y Pecuarias (INIFAP).

8. Instituto Colombiano Agropecuario (ICA). (1992). Fertilización en diversos cultivos. Quinta Aproximación. Manual de Asistencia Técnica No.25. Tibaitata, Colombia: Ediciones.

9. Ismail S. M. (2010). Influence of deficit irrigation on water use efficiency and bird pepper production (Capsicum annum L.). Meteor. Environ. Arid Land Agric. Sci. 21, 2943. Jeddah, Saudi Arabia: King Abdulaziz University.

10. Ligarreto, M.G.,Espinososa, B.N. \& Mendez, P.M. (2004). Recursos genéticos y cultivos de Ají y Pimentón (Capsicum sp.). Bogotá, Colombia: Universidad Nacional de Colombia.

11. Marouani, A. \& Harbeoui, Y. (2016). Eficiencia de nitrógeno en el cultivo de papa (Solanum tuberosum L.). Acta agronómica, 65(2), 164-169. Palmira, Colombia: Universidad Nacional de Colombia.

12. Mckean, S.J. (1993).Manual de análisis de suelos y tejido vegetal: una guía teórica y práctica de metodologías. Documento de trabajo No.129. Laboratorio de servicios analíticos. Cali, Colombia: Centro Internacional de Agricultura Tropical (CIAT).

13. Puentes, P.Y.J., Menjivar, F.J.C. y Aránzazu, H.F. (2014). Eficiencias en el uso de nitrógeno, fósforo y potasio en clones de cacao (Theobroma cacao L.). Bioagro 26:99-106.

14. Quintal, O.W.C.,Pérez, G.A., Latournerie, M.L., May, L.C., Ruíz, S.E. \& Martínez, C.A.J.(2012). Uso de agua, potencial hídrico y rendimiento de chile habanero (Capsicum chinenseJacq.). Rev. Fitotec. Mex., 35 (2), 155 -160. Chapingo, México: Sociedad mexicana de fitogenética.

15. Reyes, R.A., López, A.M., Ruíz, S.E., Latournerie, M.L., Perez, G.A., G. Lozano, C.M. \& Zavala, L.M.J. (2014). Efectividad de inoculantes microbianos en el crecimiento y productividad de chile habanero (Capsicum chinense Jacq.). Agrociencia, 48, 285-294. Chillán, Chile: Universidad de Concepción.

16. Zhu, J.H., Li, X.L., Christie, P. \& Li, J.L. (2005). Environmental implications of low nitrogen use efficiency in excessively fertilized hot pepper (Capsicum frutescens L.) cropping systems. Agriculture, Ecosystems and Environment, 111, 70-80.Belfast, UK: Queen's University.
Conflicto de Intereses

Los autores declaran no tener ningún conflicto de intereses

Recibido: 07 de abril de 2016 Aceptado: 12 de mayo de 2016 